\title{
Análise de Painéis de Alvenaria Submetidos a Recalque pelo Método dos Elementos Finitos
}

\author{
Analysis of Masonry Panels Subjected to Settlement by the Finite Element Method \\ Leonardo Teixeira da Silva ${ }^{1}$ (i) https://orcid.org/0000-0002-6015-6723 \\ Maria Fernanda Figueiredo de Oliveira ${ }^{1}$ (D) https://orcid.org/0000-0002-0258-5079 \\ ${ }^{1}$ Departamento de Estruturas e Fundações, Faculdade de Engenharia, Universidade do Estado do Rio de Janeiro, Rio de Janeiro, Brasil. \\ E-mail do autor principal: Maria Fernanda Figueiredo de Oliveira mariafer@eng.uerj.br
}

\section{Resumo}

Em construções de alvenaria estrutural, o recalque pode causar patologias severas, que podem comprometer a integridade da estrutura. No presente trabalho, o método dos elementos finitos é aplicado para simular o recalque de três painéis em alvenaria cerâmica em escala reduzida 1:3 e avaliar o surgimento de fissuras. Os painéis foram modelados com a abordagem da macromodelagem no estado plano de tensões, utilizando uma técnica de homogeneização para aproximar o comportamento constitutivo do material compósito. O objetivo principal desse estudo é investigar se essa abordagem pode fornecer resultados satisfatórios e compreender suas limitações na simulação de painéis de alvenaria submetidos a recalques. Os resultados obtidos da análise de tensões foram confrontados com os resultados experimentais dos mesmos na literatura. Através da comparação dos resultados numéricos com os valores de tensão de ruptura dos blocos, da argamassa, das juntas e da alvenaria, foi possível identificar as regiões de aparecimento de fissuras constatadas nos ensaios experimentais. A abordagem de macromodelagem com a técnica de homogeneização mostrou-se adequada para estudos preliminares.

Palavras-Chave: Alvenaria estrutural; Macromodelagem; Método dos elementos finitos;

\begin{abstract}
In structural masonry constructions, settlement can cause severe pathologies that may compromise the integrity of the whole structure. In the present study, the finite element method is applied to simulate settlement in three panels of ceramic masonry in reduced scale in order to estimate the occurrence of cracks. The panels were modeled with the macromodeling approach in the plane stress state using a homogenization technique to approximate the constitutive behavior of the composite material. The main purpose of this study is to investigate whether this approach can lead to satisfactory results and comprehend its limitations for the simulation of masonry panels subjected to settlement. The results obtained from the stress analysis were confronted with the experimental results disclosed therein in the literature. The comparison of the numerical stress values with the strength parameters of units, mortar, joints and masonry made it possible to identify the panel regions in which cracks had arisen in the experimental tests. The macromodeling approach with the homogenization technique showed to be adequate to preliminary studies.
\end{abstract}

Key-words: Structural masonry; Macromodeling; Finite element method. 


\section{Introdução}

$\mathrm{Na}$ última década, a técnica construtiva em alvenaria estrutural tem sido escolhida por diversas construtoras brasileiras por mostrar-se bastante eficiente, já que proporciona redução de custos quando comparada às técnicas tradicionais [1]. No Brasil, as normas técnicas que regulamentam projetos em alvenaria estrutural de blocos cerâmicos e de concreto são NBR 15961-1 e 2 [2,3] e NBR 15812-1 e $2[4,5]$. No entanto, há ainda muitos aspectos que merecem estudo e aperfeiçoamento.

Com o avanço das pesquisas, o estudo do comportamento de estruturas em alvenaria estrutural tornou-se mais sofisticado e menos empírico. A modelagem numérica de estruturas em alvenaria é utilizada para diversos fins, como para a obtenção do caminho de tensões na fase de projeto, para a avaliação da segurança e resistência de edificações quando houver mudança na usabilidade, ocorrência de cargas de impacto e recalque $[6,7,8,9]$, e para a identificação de causas de patologia e sugestões de reparos adequados $[\mathbf{1 0}, \mathbf{1 1}]$.

Com relação à simulação numérica, os desafios são devidos ao comportamento complexo do material, que pode ser influenciado pela anisotropia, pelas propriedades mecânicas dos blocos e das juntas de assentamento, pelo arranjo geométrico desses componentes bem como pela mão-de-obra utilizada no processo de construção [12].

A alvenaria é um material heterogêneo e anisotrópico, com resistência à compressão elevada devido às unidades de pedra, cerâmica ou concreto; mas com baixa resistência à tração, devido à adesão entre o bloco e a argamassa. É um material semifrágil, com comportamento não linear das juntas e com diversos mecanismos de ruptura - fissura nas juntas, escorregamento nas juntas, fissura das unidades e fendilhamento [13]. O comportamento do material é também dependente da escala em que se analisa o problema, como observado em diversos experimentos.

Pela complexidade do comportamento da alvenaria estrutural, as abordagens de modelagem são distintas a depender da escala utilizada $[\mathbf{1 4}, \mathbf{1 5}, \mathbf{1 6}, \mathbf{1 7}]$ :

Macromodelagem: $O$ bloco, a argamassa e a junta são considerados dispersos no meio contínuo e toda a alvenaria é considerada com propriedades homogêneas. Os dados de entrada são menores que os exigidos para a micromodelagem, com a vantagem de realizar análise em menor tempo e esforço computacional reduzido. As propriedades do material equivalente podem ser obtidas através de ensaios experimentais ou de técnicas de homogeneização [18]. Essa abordagem é apropriada quando se tem interesse no comportamento global da estrutura;

Micromodelagem simplificada: Considera-se 0 comportamento do bloco e da argamassa como um só material, por elementos contínuos com dimensões expandidas. A interface bloco/argamassa é representada por elementos descontínuos;

Micromodelagem detalhada: Considera-se 0 comportamento de cada material que compõe a alvenaria separadamente: os blocos e a argamassa são representadas por elementos contínuos e a interface bloco/argamassa é representada por elementos descontínuos. Essa abordagem é mais apropriada quando se deseja obter resultados localizados.

Quanto mais sofisticada a abordagem de modelagem adotada, maior a quantidade de dados de entrada referentes ao comportamento dos materiais e maior o custo computacional.

Dentre as patologias em alvenaria estrutural, as causadas por recalques estão entre as mais severas. Por ser constituída de material compósito de pequena ductilidade, a alvenaria estrutural pode apresentar fissuração suficiente para comprometer a integridade de toda a estrutura. Esse trabalho investiga a aplicação da abordagem de macromodelagem para avaliar o surgimento de fissuras em painéis de alvenaria pelo métodos dos elementos finitos.

Os painéis em alvenaria cerâmica escolhidos para estudo foram ensaiados em laboratório, em escala reduzida, por Holanda Jr. [19], que mapeou o aparecimento de fissuras e realizou a caracterização física dos blocos, da argamassa, das juntas e da parede. Além disso, Holanda Jr. [19] analisou numericamente e experimentalmente os deslocamentos e sua relação com o aparecimento de fissuras em paredes de alvenaria submetidas à carregamento de compressão e recalques.

No presente trabalho, programa ANSYS [20] foi utilizado para modelar os painéis com a abordagem da macromodelagem no estado plano de tensões, utilizando uma técnica de homogeneização para aproximar o comportamento constitutivo do material compósito. O campo de tensões obtido nas análises 
numéricas do presente estudo foi confrontado com o mapeamento de fissuras obtido experimentalmente.

\section{Geometria, Propriedades dos Materiais e Carregamento dos Painéis de Alvenaria Analisados}

O presente trabalho simulou computacionalmente dois painéis de alvenaria cerâmica em escala reduzida 1:3: um painel sem abertura e um painel com aberturas de porta e janela. Os referidos painéis foram ensaiados em laboratório por Holanda Jr. [19], que investigou as configurações deformadas e a fissuração dos mesmos quando submetidos a uma carga de compressão e recalques de apoio. Os painéis foram construídos sobre vigas de altura de $18 \mathrm{~cm}$ com três apoios igualmente espaçados, como ilustrado na Figura 1.

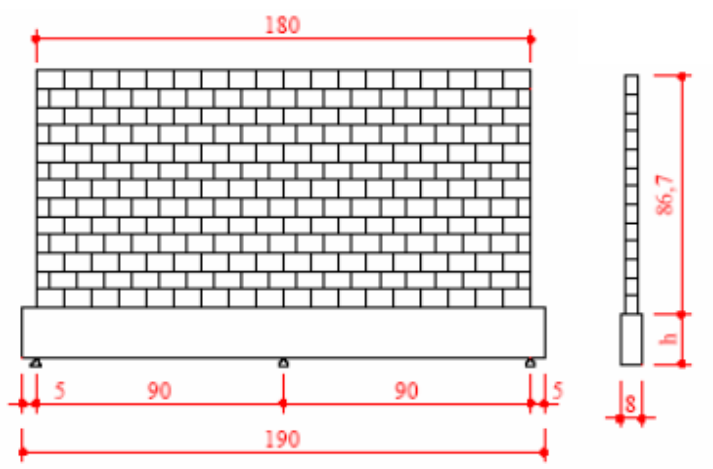

(a)

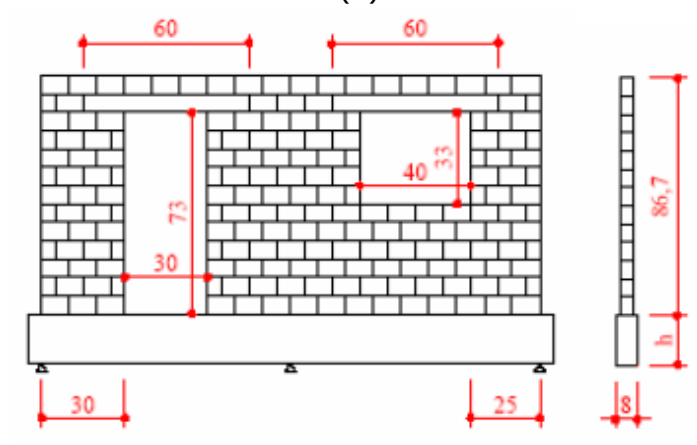

(b)

Figura 1: Painéis analisados (dimensões em $\mathrm{cm}$ ): (a) sem abertura e (b) com aberturas de porta e janela.

Fonte: Holanda Jr. (2002, p. 42).

Os parâmetros de deformabilidade (Quadro 1) e de resistência (Quadro 2) dos materiais que compõem a alvenaria foram obtidos por Holanda Jr. [19] através de ensaios experimentais. Os valores de coeficiente de Poisson do bloco cerâmico e da argamassa foram 72 adotados a partir de valores utilizados num estudo sobre homogeneização feito por Barreto [21].

O carregamento foi aplicado conforme o ensaio experimental realizado. Inicialmente, os painéis foram submetidos a um carregamento de compressão vertical, aplicado de modo distribuído ao longo do topo do painel (Quadro 3). Em seguida, o recalque foi aplicado no apoio central de cada painel até que as reações correspondentes se anulassem.

Quadro 1: Parâmetros de deformabilidade dos blocos, das juntas/argamassa, da alvenaria e do concreto das vigas e vergas: Módulo de elasticidade longitudinal $(E)$ e coeficiente de Poisson $(v)$.

\begin{tabular}{|c|c|}
\hline Material & Parâmetro \\
\hline Bloco cerâmico & $\begin{array}{l}E=1055,4 \mathrm{kN} / \mathrm{cm}^{2} \\
v=0,1\end{array}$ \\
\hline Argamassa/juntas & $\begin{aligned} E & =1527,0 \mathrm{kN} / \mathrm{cm}^{2} \\
v & =0,2\end{aligned}$ \\
\hline Alvenaria & $\begin{array}{l}E=647,9 \mathrm{kN} / \mathrm{cm}^{2} \\
v=0,1\end{array}$ \\
\hline Concreto da viga & $\begin{aligned} E & =1862,0 \mathrm{kN} / \mathrm{cm}^{2} \\
v & =0,2\end{aligned}$ \\
\hline Concreto das vergas & $\begin{array}{l}E=2706,0 \mathrm{kN} / \mathrm{cm}^{2} \\
v=0,2\end{array}$ \\
\hline
\end{tabular}

Fonte: Valores obtidos de Holanda Jr. (2002) e Barreto (2002).

Quadro 2: Parâmetros de resistência dos blocos, das juntas/argamassa e da alvenaria: Tensão normal de ruptura à compressão $\left(\sigma_{R C}\right)$ e tensão cisalhante de ruptura $\left(\tau_{R}\right)$

\begin{tabular}{|c|c|}
\hline Material & \multicolumn{1}{c|}{ Parâmetro } \\
\hline Bloco cerâmico & $\sigma R C=2,93 \mathrm{kN} / \mathrm{cm}^{2}$ \\
\hline Argamassa $/$ juntas & $\begin{array}{l}\sigma R C=1,22 \mathrm{kN} / \mathrm{cm}^{2} \\
\tau_{\mathrm{R}}=0,06-0,75 \sigma_{c}(\mathrm{MPa})\end{array}$ \\
\hline Alvenaria & $\begin{array}{l}\sigma R C=1,19 \mathrm{kN} / \mathrm{cm}^{2} \\
\tau_{R}=0,059 \mathrm{kN} / \mathrm{cm}^{2}\end{array}$ \\
\hline
\end{tabular}

Fonte: Valores obtidos de Holanda Jr. (2002).

Quadro 3: Carga de compressão total aplicada no topo do painel como carregamento distribuído.

\begin{tabular}{|c|c|}
\hline \multicolumn{1}{|c|}{ Painel } & \multicolumn{1}{c|}{ Carga total } \\
\hline Sem abertura & $167 \mathrm{kN}$ \\
\hline
\end{tabular}

http: / / dx.doi.org/10.25286/repa.v4i1.927 


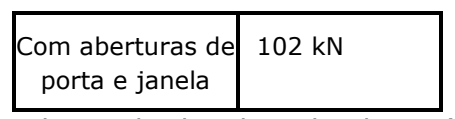

Fonte: Valores obtidos de Holanda Jr. (2002).

\section{Modelagem pelo Método dos Elementos Finitos}

Os painéis foram modelados no programa ANSYS [20] no estado plano de tensões com elementos quadrilaterais lineares do tipo PLANE42, mantendo a malha proposta por Holanda Jr. [19]. As Figuras 2 e 3 ilustram as malhas de elementos finitos empregadas.

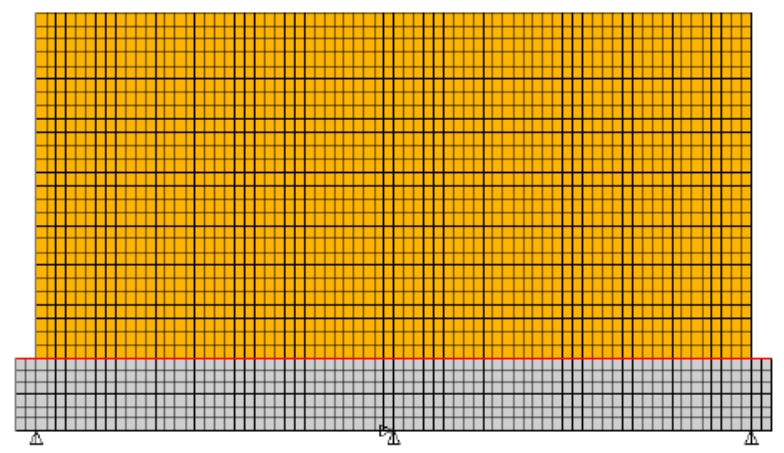

Figura 2: Malha de elementos finitos do painel sem abertura.

Fonte: Adaptação de Holanda Jr. (2002, p. 158).

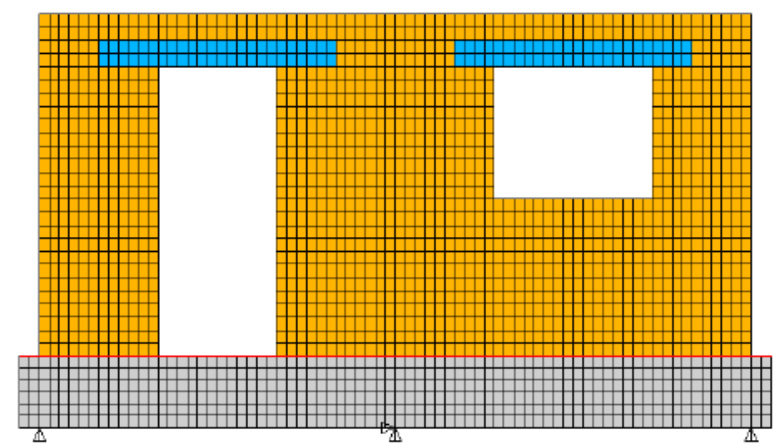

Figura 3: Malha de elementos finitos do painel com aberturas e porta e janela.

Fonte: Adaptação de Holanda Jr. (2002, p. 158).

A alvenaria foi modelada como material linear elástico isotrópico, sendo necessário aplicar uma técnica de homogeneização para se obter os valores dos parâmetros de deformabilidade equivalentes. Empregou-se a técnica de homogeneização para material transversalmente isotrópico proposta por Pande et al. [22], que está descrita de forma detalhada no trabalho de Barreto [21]. Nessa técnica, adotam-se as hipóteses de alinhamento perfeito das juntas verticais e aderência perfeita entre blocos e argamassa. A partir das dimensões geométricas do arranjo da alvenaria e dos parâmetros de deformabilidade do bloco e da argamassa, considerando a isotropia também na direção vertical, chega-se aos valores de propriedades para o material homogêneo apresentados no Quadro 4.

Quadro 4: Parâmetros de deformabilidade da alvenaria pela aplicação de técnica de homogeneização: Módulo de elasticidade longitudinal $(E)$ e coeficiente de Poisson $(v)$.

\begin{tabular}{|c|l|}
\hline Material & \multicolumn{1}{c|}{ Parâmetro } \\
\hline Alvenaria & $\begin{array}{l}E=1079,18 \mathrm{kN} / \mathrm{cm}^{2} \\
v=0,107\end{array}$ \\
\hline
\end{tabular}

Fonte: O autor.

\section{Resultados e Discussões}

Para cada painel analisado, os resultados de tensões obtidos numericamente foram verificados quanto às seguintes possibilidades de ruptura:

Compressão no bloco: A tensão $\sigma_{y}$ foi confrontada com a tensão de ruptura à compressão do bloco $\left(\sigma_{R C}=2,93 \mathrm{kN} / \mathrm{cm}^{2}\right)$;

Compressão na argamassa: As tensões $\sigma_{x}$ (juntas verticais) e $\sigma_{y}$ (juntas horizontais) foram confrontadas com as tensões de ruptura à compressão da argamassa $\left(\sigma_{R C}=1,22 \mathrm{kN} / \mathrm{cm}^{2}\right)$;

Tração nas juntas: As tensões $\sigma_{x}$ (juntas verticais) e $\sigma_{y}$ (juntas horizontais) foram confrontadas com valores nulos de tensão. Dessa forma, admitiu-se que as juntas sofrem separação quando submetidas à tração;

Cisalhamento nas juntas: $A$ tensão $\tau_{x y}$ foi confrontada com a envoltória de ruptura de MohrColumb $\tau_{R}=0,6-0,75 \sigma_{c} \quad(\mathrm{MPa})$, sendo que $\sigma_{c}$ assume valores de $\sigma_{x}$ e $\sigma_{y}$, para juntas verticais e horizontais, respectivamente. Deste modo, regiões com ruptura podem ser indicadas pelo parâmetro RATIO $=\frac{\tau_{x y}}{\tau_{\text {rup }}}>1$;

Compressão axial da alvenaria: A tensão $\sigma_{y}$ foi confrontada com a tensão de ruptura à compressão da alvenaria $\left(\sigma_{R C}=1,19 \mathrm{kN} / \mathrm{cm}^{2}\right)$; 
Compressão diagonal da alvenaria: A tensão $\tau_{\max }$ foi confrontada com a tensão de ruptura ao cisalhamento da alvenaria $\left(\tau_{R}=0,059 \mathrm{kN} / \mathrm{cm}^{2}\right)$.

Os resultados dessa análise de tensões foram confrontados com o mapeamento de fissuras obtido experimentalmente por Holanda Jr. [19], possibilitando identificar o tipo de falha para cada região fissurada dos painéis.

\subsection{Painel Sem Aberturas}

A Figura 4 mostra a distribuição das tensões principais e permite avaliar o caminho das cargas para o painel ensaiado: bielas comprimidas nas diagonais que partem dos apoios externos e regiões tracionadas na base do painel.

As Figuras 5, 6 e 7 mostram a distribuição das tensões $\sigma_{y}, \tau_{x y}$ (RATIO) e $\tau_{\text {max }}$, respectivamente. A comparação com valores de resistência permite identificar as regiões de ruptura A, B, C e D (Figura 8) e suas respectivas causas, resumidas no Quadro 5.

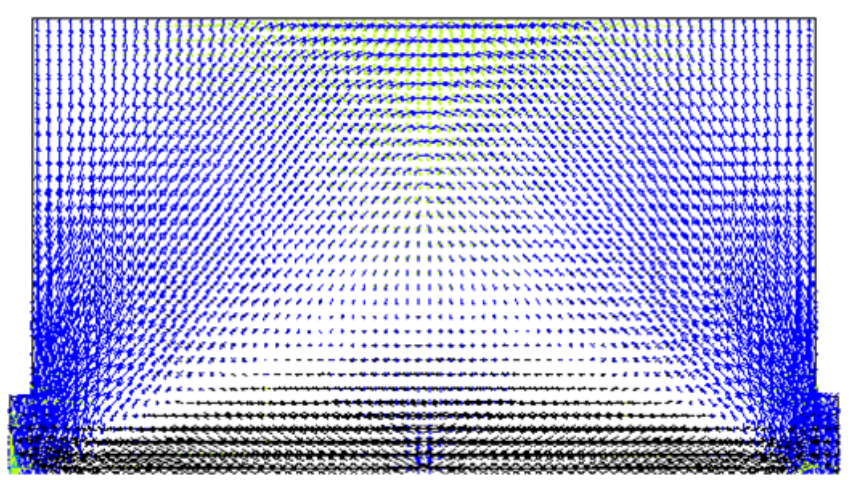

Figura 4: Tensões principais para o painel sem aberturas: $\sigma_{1}$ (azul), $\sigma_{2}$ (verde) e $\sigma_{3}$ (preto). Fonte: $\mathrm{O}$ autor.

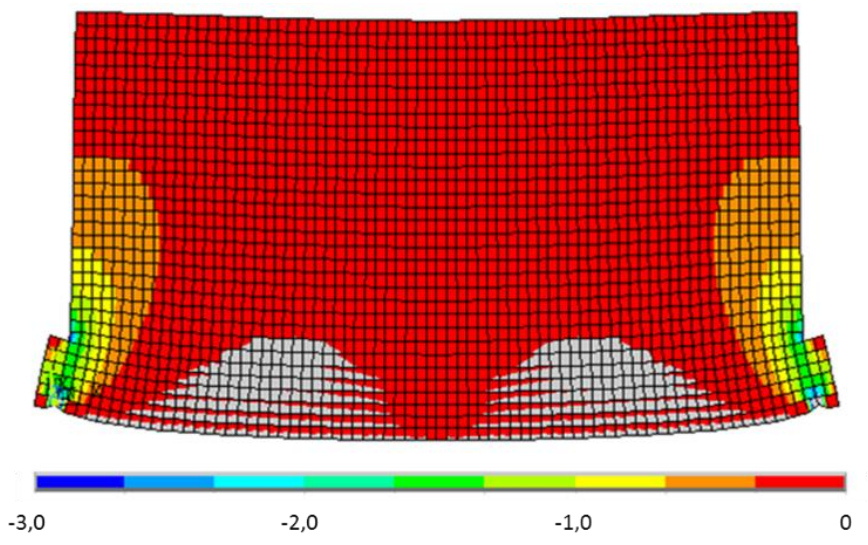

Figura 5: Valores de tensão $\sigma_{y}\left(\mathrm{kN} / \mathrm{cm}^{2}\right)$ indicando a ruptura nas regiões A e B (regiões em cinza tracionadas). Fonte: $\mathrm{O}$ autor.

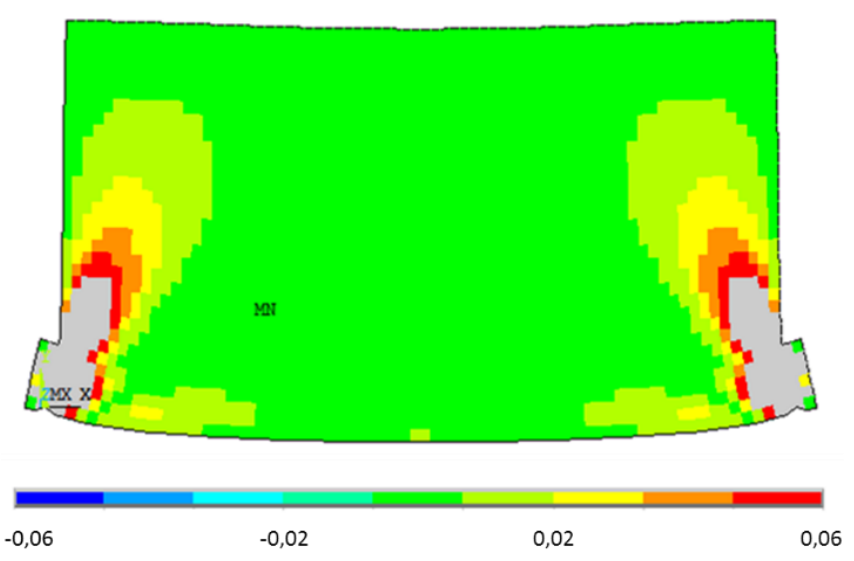

Figura 6: Valores de tensão cisalhante máxima $\tau_{\max }$ $\left(\mathrm{kN} / \mathrm{cm}^{2}\right)$ indicando a ruptura nas regiões A e C (regiões em cinza com tensões que superam a resistência). Fonte: O autor. 


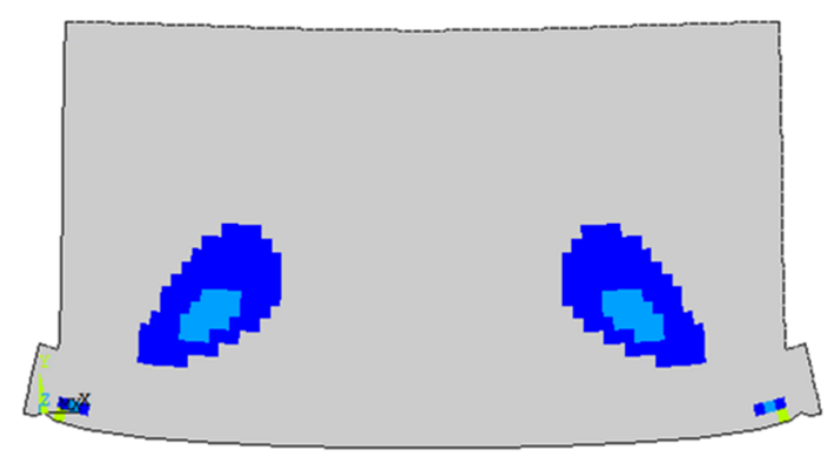

(a)

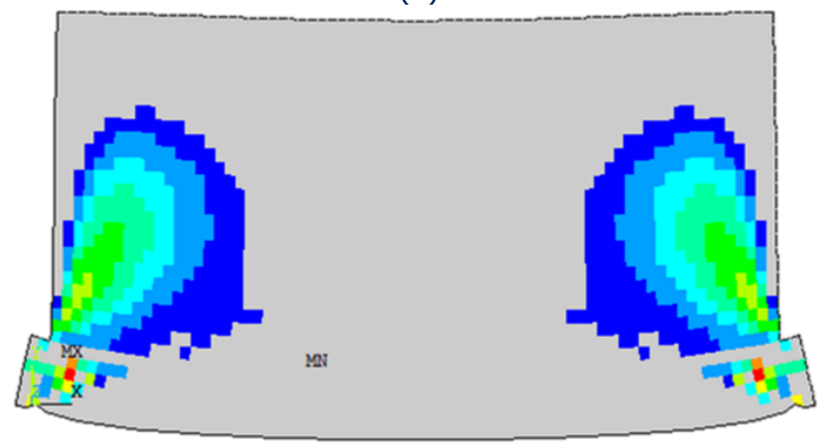

(b)

1,0 1,7

2,3

Figura 7: Valores de RATIO $>1$ indicando a ruptura das juntas horizontais (a) e verticais (b) nas regiões C e D (regiões em cinza com RATIO $<1$ ).

Fonte: $\mathrm{O}$ autor.

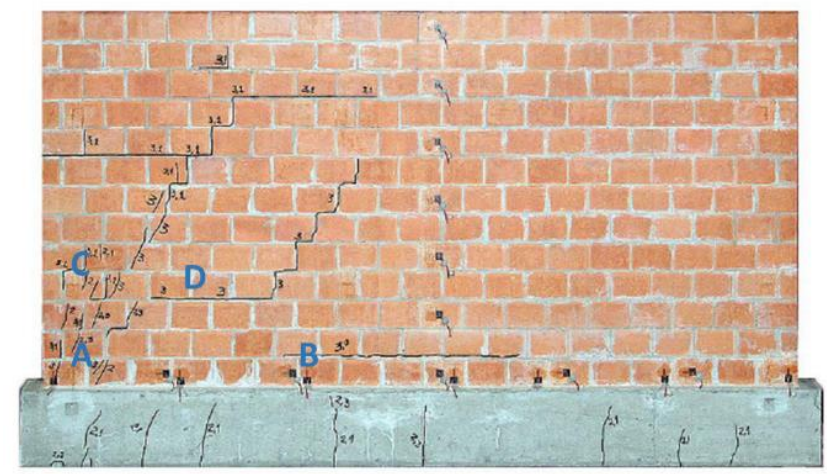

Figura 8: Regiões que apresentaram ruptura na análise numérica para o painel sem aberturas $(A, B, C, D)$. Fonte: Adaptação de Holanda Jr. (2002, p. 82).
Quadro 5: Tipo de ruptura indicado pela análise numérica para o painel sem aberturas

\begin{tabular}{|c|l|}
\hline Região & \multicolumn{2}{c|}{ Tipo de ruptura } \\
\hline A & $\begin{array}{l}\text { Compressão axial da alvenaria e da argamassa } \\
\text { Cisalhamento nas juntas verticais } \\
\text { Cisalhamento na alvenaria }\end{array}$ \\
\hline B & Tração nas juntas horizontais \\
\hline C & $\begin{array}{l}\text { Cisalhamento nas juntas verticais } \\
\text { Cisalhamento na alvenaria }\end{array}$ \\
\hline D & Cisalhamento nas juntas horizontais e verticais \\
\hline
\end{tabular}

Fonte: O autor.

$\mathrm{Na}$ análise numérica, devido à simetria do problema, os resultados indicam regiões de ruptura em ambos os lados do painel. No ensaio experimental, apenas o lado esquerdo do painel (Figura 8) apresentou fissuração, provavelmente por motivo de assimetria do problema real, seja geométrica, de carregamento ou no comportamento do material. Apesar dessa diferença nos resultados, a análise que se segue considera que é ainda válido identificar as causas da ruptura do painel ensaiado a partir dos resultados numéricos.

Na região $A$, os resultados de tensão axial da Figura 5 indicam ruptura por compressão da argamassa e da alvenaria, concordando com o fissuramento vertical dos blocos e da argamassa observado no painel ensaiado. Os resultados de tensão axial também acusam ruptura por tração das juntas horizontais na região $B$, que pode ser observada pela extensa fissura horizontal entre a primeira e a segunda fiada do painel ensaiado.

Nas regiões $A, C$ e $D$, os valores de tensão de cisalhamento das Figuras 6 e 7 indicam ruptura, seja na alvenaria ou nas juntas horizontais e verticais. Esses resultados estão coerentes com o padrão de fissuração encontrado no painel ensaiado, que apresenta fissuras diagonais nos blocos e argamassa das regiões $A$ e $C$ bem como fissuras em padrão ziguezague nas juntas das regiões $C$ e $D$.

\subsection{Painel com Aberturas de Porta e Janela}

A Figura 9 mostra a distribuição das tensões principais e permite avaliar o caminho das cargas para 
o painel ensaiado: bielas comprimidas nas diagonais que partem dos apoios externos, mas desviando das aberturas; regiões tracionada na base do painel e em algumas regiões próximas às aberturas. Verifica-se também concentrações de tensões próximas aos cantos das aberturas, relacionadas com patologias em painéis com aberturas $[\mathbf{2 3}, \mathbf{2 4}]$.

As Figuras 10, 11 e 12 mostram a distribuição das tensões $\sigma_{y}, \sigma_{x}$ e $\tau_{x y}$ (RATIO), respectivamente. A comparação com valores de resistência permite identificar as regiões de ruptura A, B, C e D (Figura 13) e suas respectivas causas, resumidas no Quadro 6.

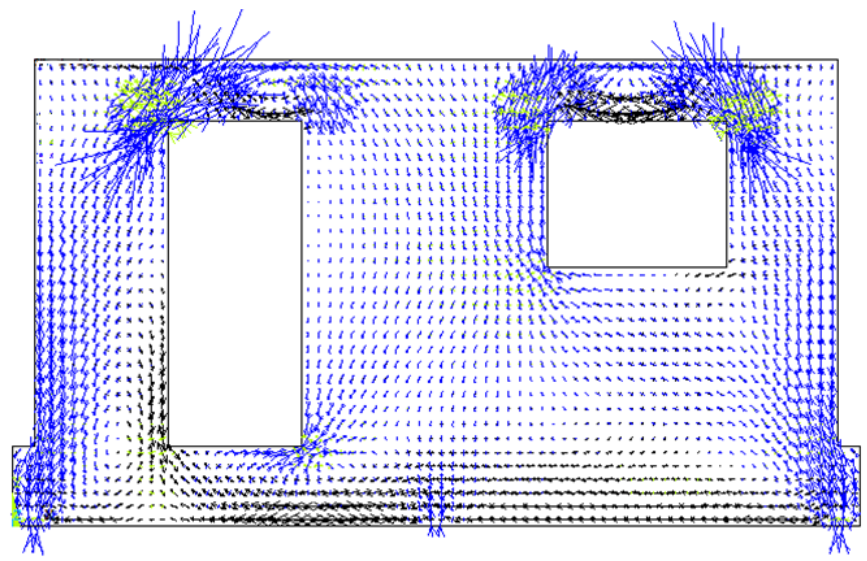

Figura 9: Tensões principais para o painel com aberturas de porta e janela: $\sigma_{1}$ (azul), $\sigma_{2}$ (verde) e $\sigma_{3}$ (preto). Fonte: O autor.

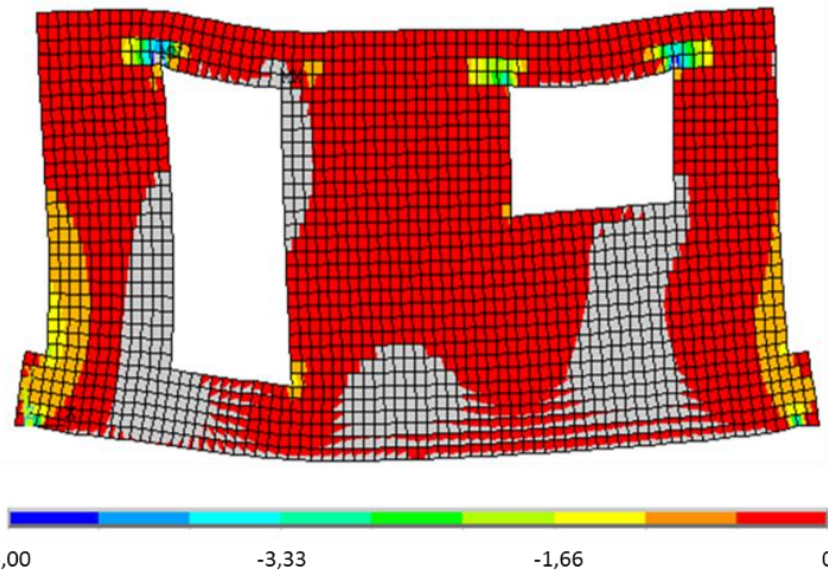

Figura 10: Valores de tensão $\sigma_{y}\left(\mathrm{kN} / \mathrm{cm}^{2}\right)$ indicando a ruptura nas regiões $B, C$ e $D$ (regiões em cinza tracionadas).

Fonte: O autor.

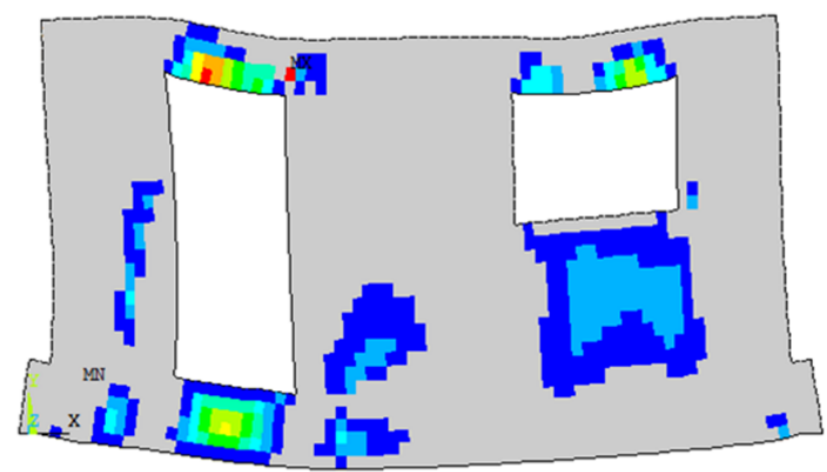

(a)

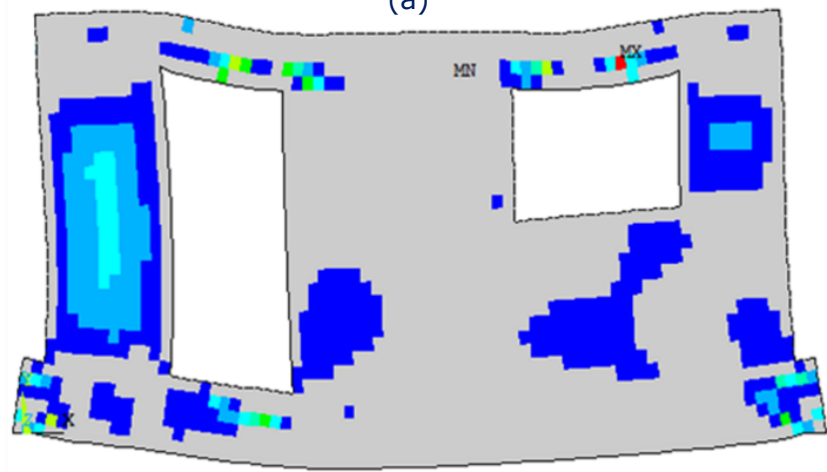

(b)

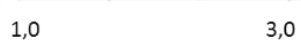

5,0

Figura 11: Valores de RATIO $>1$ indicando a ruptura das juntas horizontais (a) e verticais (b) nas regiões $B$ e $C$ (regiões em cinza com RATIO $<1$ ).

Fonte: O autor.

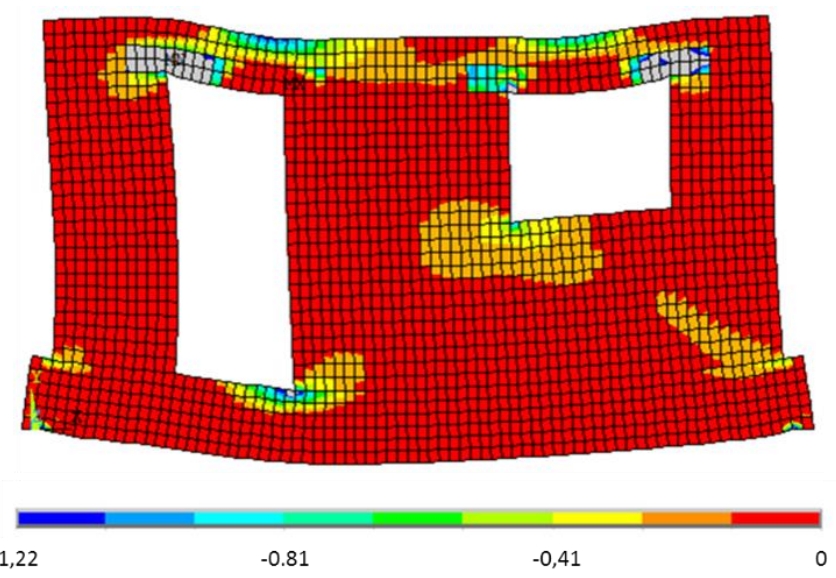

Figura 12: Valores de tensão $\sigma_{x}\left(\mathrm{kN} / \mathrm{cm}^{2}\right)$ indicando a ruptura na região $A$ (regiões em cinza valores de tensão inferiores a $-1,22 \mathrm{kN} / \mathrm{cm}^{2}$ ).

Fonte: $\mathrm{O}$ autor. 


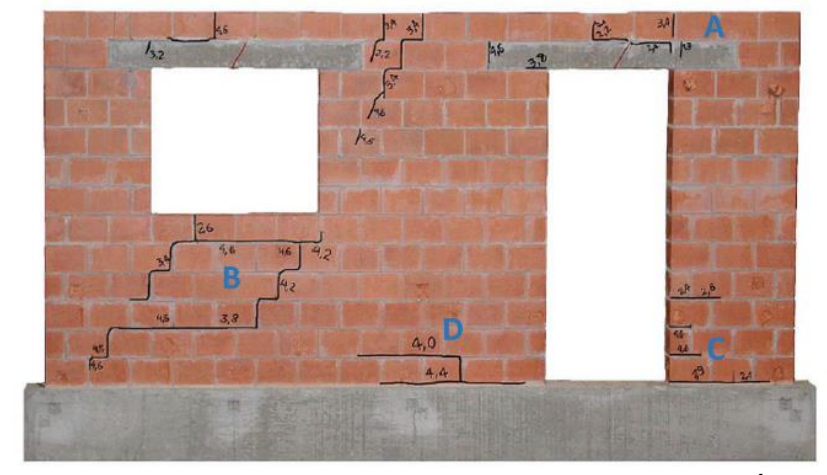

Figura 13: Regiões que apresentaram ruptura na análise numérica para o painel com aberturas de porta e janela $(A, B, C, D)$.

Fonte: Adaptação de Holanda Jr. (2002, p. 118).

Quadro 6: Tipo de ruptura indicado pela análise numérica para o painel com aberturas de porta e janela.

\begin{tabular}{|c|c|}
\hline Região & Tipo de ruptura \\
\hline A & $\begin{array}{l}\text { Compressão da argamassa nas juntas verticais } \\
\text { Cisalhamento nas juntas horizontais e verticais }\end{array}$ \\
\hline B & $\begin{array}{l}\text { Tração nas juntas horizontais } \\
\text { Cisalhamento nas juntas horizontais e verticais }\end{array}$ \\
\hline $\mathrm{C}$ & $\begin{array}{l}\text { Tração nas juntas horizontais } \\
\text { Cisalhamento nas juntas horizontais }\end{array}$ \\
\hline D & Tração nas juntas horizontais \\
\hline
\end{tabular}

Os resultados de tensão axial da Figura 10 indicam ruptura por tração das fiadas nas regiões $B, C$ e $D$, que pode ser verificada no painel ensaiado pelo aparecimento de fissuras horizontais de maior extensão nessas regiões, tanto entre fiadas como entre a fiada inicial e a viga. A distribuição de tensão normal na direção horizontal da Figura 12 acusa valores elevados acima das vergas da porta e da janela (região A), podendo levar à ruptura por compressão das juntas verticais, concordando assim com o aparecimento das fissuras verticais na argamassa nessas regiões.

Nas regiões A, B e C, os valores de tensão de cisalhamento da Figura 11 indicam também ruptura nas juntas horizontais e verticais. Esses resultados estão coerentes com o padrão de fissuração em padrão ziguezague encontrado na região $B$ do painel ensaiado, podendo também ter contribuído para as fissuras nas regiões $A$ e $C$.

A partir dos resultados de tensão obtidos da modelagem numérica realizada, não foi possível identificar a causa da ruptura na porção superior do painel, próximo à janela. Pelo padrão de fissuramento diagonal nos blocos e argamassa, pode-se identificar a ruptura por cisalhamento do alvenaria.

\section{Conclusões}

O presente estudo aplicou a abordagem da macromodelagem com 0 método dos elementos finitos para analisar o comportamento de painéis de alvenaria em modelo reduzido submetidos a cargas de compressão e recalque. Os parâmetros de propriedade mecânicas dos materiais foram obtidos por ensaios laboratoriais em Holanda Jr. [19] e possibilitaram a aplicação de uma técnica de homogeneização para a obtenção dos parâmetros de deformabilidade elástica da alvenaria. Os resultados das análises numéricas de tensões foram confrontados com os dados experimentais dos painéis ensaiados.

Apesar da abordagem de macromodelagem capturar apenas o comportamento global da estrutura e de se ter adotado um comportamento homogêneo linear elástico para a alvenaria, foi possível identificar várias regiões de fissuração pela análise das tensões frente às tensões de ruptura dos blocos, das juntas e da alvenaria como um todo. Os resultados de tensões principais também mostraram um bom indicativo do caminho das cargas nos painéis, podendo ser usados para orientar a fase de projeto.

Deve-se observar que a macromodelagem empregada considerou o material compósito como elástico linear e que a verificação de ocorrência de fissuração foi obtida pela comparação com critérios de ruptura apenas na etapa final do carregamento. Como a redistribuição de tensões devido à fissuração não foi modelada, o desenvolvimento progressivo de fissuras também não pôde ser capturado na análise. Os resultados obtidos pela macromodelagem podem ainda ser melhorados com a aplicação de modelos constitutivos e critérios de ruptura mais sofisticados para a alvenaria no estado biaxial de tensões $[25,26]$.

Apesar das limitações, esses resultados podem ser usados para sinalizar e guiar mudanças para o aperfeiçoamento do modelo numérico em aspectos como o refinamento da malha, a escolha de modelos constitutivos mais adequados e até a necessidade de mudança de abordagem, a depender do tipo de 
resultado em que se tem interesse. Os resultados da macromodelagem também podem auxiliar na identificação de parâmetros dos materiais que precisam de melhor caracterização quanto à deformabilidade e resistência do material compósito.

\section{Referências}

[1] RAMALho, M. A.; CORRÊA, M. R. S. Projeto de Edifícios de Alvenaria Estrutural. São Paulo: Pini, 2003.

[2] ASSOCIAÇÃO BRASILEIRA DE NORMAS TÉCNICAS. NBR 15961: Alvenaria estrutural: Blocos de concreto - Parte 1: Projeto. Rio de Janeiro: ABNT, 2011.

[3] ASSOCIAÇÃO BRASILEIRA DE NORMAS TÉCNICAS. NBR 15961: Alvenaria estrutural: Blocos de concreto - Parte 2: Execução e controle de obras. Rio de Janeiro: ABNT, 2011.

[4] ASSOCIAÇÃO BRASILEIRA DE NORMAS TÉCNICAS. NBR 15812: Alvenaria estrutural: Blocos cerâmicos - Parte 1: Projeto. Rio de Janeiro: ABNT, 2010.

[5] ASSOCIAÇÃO BRASILEIRA DE NORMAS TÉCNICAS. NBR 15812: Alvenaria estrutural: Blocos cerâmicos - Parte 2: Execução e controle de obras. Rio de Janeiro: ABNT, 2010.

[6] ASTERIS, P. G.; PLEVRIS, V. Handbook of research on seismic assessment and rehabilitation of historic structures. Advances in Civil and Industrial Engineering Book Series. Engineering Science Reference, 2015.

[7] HAO, H. Numerical modelling of masonry wall response to blast loads. Australian Journal of Structural Engineering, v. 10, n. 1, p. 37-52, 2009.

[8] IDRIS, J.; AL-HEIB, M.; VERDEL, T. Numerical modelling of masonry joints degradation in built tunnels. Tunnelling and Underground Space Technology, v. 24, p. 617-626, 2009.

[9] LIU, G.; HOULSBY, G. T.; AUGARDE, C. E. 2dimensional analysis of settlement damage to masonry buildings caused by tunneling. The Structural Engineer, v. 79, p. 19-25, 2000.
[10] GAROFANO, A.; CERONI, F.; PECCE, M. Modelling of the in-plane behaviour of masonry walls strengthened with polymeric grids embedded in cementitious mortar layers. Composit Part B, v. 85, p. 243-258. 2016.

[11] THEODOSSOPOLOS, D.; SINHA, B. A review of analytical methods in the current design processes and assessment of performance of masonry structures. Construction and Building Materials, v. 41, p. 990-1001, 2013.

[12] PELETEIRO, S. C. Contribuições à Modelagem Numérica de Alvenaria Estrutural. Tese de doutorado. Escola de Engenharia de São Carlos, São Carlos, 2002. 143 p.

[13] LOURENÇO, P. B.; ROTS, J. G.; BLAAUWENDRAAD, J. Two approaches for the analysis of masonry structures: micro and macromodeling. HERON, v. 40, p. 313-340, 1995.

[14] LOURENÇO, P. B. Um micro-modelo para a análise de estruturas de alvenaria. Engenharia Civil UM, n. 3, p. 15-28, 1996.

[15] LOURENÇO, P. B. Computational strategies for masonry structures: Multi-scale modelling, dynamics, engineering applications and other challenges. In: Congresso de Métodos Numéricos en Ingeniería. 2013, Bilbao. Actas del Congreso de Métodos Numéricos em Ingeniería, Bilbao: 2013. p. 1-17.

[16] GIORDANO, A.; MELE, E.; DE LUCA, A. Modelling of historical masonry structures: comparison of different approaches through a case study. Engineering Structures, v. 24, p. 10571069, 2002.

[17] SHIEH-BEYGI, B.; PIETRUSZCZAK, S. Numerical analysis of structural masonry: mesoscale approach. Computers and Structures, v. 86, p. $1958-1973,2008$.

[18] REKIK, A.; LEBON, F. Homogenization methods for interface modeling in damaged masonry. Advances in Engineering Software, v. 46, p. 35-42, 2012.

[19] HOLANDA JR., O. G. Influência de Recalques em Edifícios de Alvenaria Estrutural. Tese de Doutorado. Escola de Engenharia de São Carlos, 
Universidade de São Paulo, São Paulo, 2002. $224 \mathrm{p}$.

[20] ANSYS - Swanson Analysis Systems, 2016 SAS IP, Inc. Southpoint, 2600 ANSYS Drive, Canonsburg, PA 15317. Release 17.2, ANSYS Academic Research Mechanical, 2016.

[21] BARRETO, A. S. P. Análise Numérica de Painéis de Alvenaria Estrutural utilizando Técnica de Homogeneização. Dissertação de Mestrado. Universidade Federal de Pernambuco, Recife, 2002. 148 p.

[22] PANDE, G. N.; LIANG, X.; MIDDLETON, J. Equivalent elastic moduli for brick masonry. Computers and Geotechnics, v. 8, p. 243-265, 1989.

[23] BAUER, R. J. F. Patologias em alvenaria estrutural de blocos vazados de concreto. Caderno Técnico Alvenaria Estrutural, Revista Prima, CT5, p. 33-38, São Paulo: Ed. Mandarim Ltda, 2008.

[24] SAMPAIO, M. B. Fissuras em Edifícios Residenciais em Alvenaria Estrutural. Dissertação de Mestrado. Escola de Engenharia de São Carlos, Universidade de São Paulo, São Paulo, 2010. $104 \mathrm{p}$.

[25] SYRMAKEZIS, C. A.; ASTERIS, P. G. Masonry failure criterion under biaxial stress state. Journal of Materials in Civil Engineering, v. 13, p. 58-64, 2001.

[26] PAGE, A. W. The biaxial compressive strength of brick masonry. Proceedings of the Institution of Civil Engineers, Parte 2, v. 71, p. 893-906, 1981. 